\title{
ANGULAR INTEGRATION USING SYMBOLIC STATE EXPANSIONS
}

\author{
C. Froese Fischer ${ }^{\mathrm{a}}$ and D. Ellis ${ }^{\mathrm{b}}$ \\ ${ }^{a}$ Department of Computer Science, Box 1679B, Vanderbilt University, Nashville, TN 37235, USA \\ E-mail: Charlotte.F.Fischer@Vanderbilt.Edu \\ ${ }^{\mathrm{b}}$ Department of Physics and Astronomy, University of Toledo, Toledo, $\mathrm{OH} 43606$, USA
}

Received 23 April 2004

Dedicated to the 100th anniversary of Professor A. Jucys

\begin{abstract}
Angular integrations are not concerned with the principal quantum numbers of orbitals, only their angular momentum coupling, although antisymmetry and normalization need to be considered. We show that by expressing wave function expansions in terms of certain symbolic states, the angular data needed for a calculation becomes largely independent of problem size under the assumption that one- and two-electron operators for matrix elements between two-electron states can be evaluated when needed. Furthermore, for the Coulomb interaction, all many-electron matrix elements between symbolic states arising from single and double excitations from a multireference set can be expressed in terms of a sum of contributions, each of which is a product of three factors: a constant, a two-electron matrix element, and a factor depending on the orbital quantum numbers of the interacting orbitals. The $1 s^{2} 2 s^{2}{ }^{1} S$ beryllium ground state is considered in detail.
\end{abstract}

Keywords: angular integration, symbolic state expansion, Coulomb interaction, matrix elements

PACS: $31.15 .-\mathrm{p}, 02.70 .-\mathrm{c}$

\section{Introduction}

The complexity of the "Fock equations" (as Hartree called them) presented too large a challenge for a numerical solution at the time when they appeared in 1930. Instead, papers began to appear that used Slater's theory for energy expressions [1] along with Hartree's self-consistent field radial functions from which all the needed integrals could be evaluated. It soon became evident that such methods would not have the correct ratios for energy differences between terms of a configuration. Theory predicted that for terms of $p^{2}$ or $p^{4}$ the ratio $\left({ }^{1} D-{ }^{1} S\right) /\left({ }^{3} P-{ }^{1} D\right)$ would have a value of 1.5 but in $\mathrm{O}^{+2}$ the observed ratio is 1.04 . Could it be the effect of exchange on the wave function (which turned out to be negligible) or the effect of what is now called configuration interaction (CI) but was referred to by Hartree et al. [2] as superposition of configurations? In their 1939 paper, they explore the effect of exchange and the superposition of $2 s^{2} 2 p^{n}$ and $2 p^{n+2}$ on wave functions for $\mathrm{O}, \mathrm{O}^{+}$, and $\mathrm{O}^{+2}$. The ratios were still not in good agreement with observation, partly because of an error in a coefficient and partly because far more correlation is needed to get this ratio of energy differences correct.
The main computational difficulty was associated with the evaluation of the Slater integrals, particularly the $Y^{k}$ function,

$$
Y^{k}\left(n l, n^{\prime} l^{\prime} ; r\right)=r \int_{0}^{\infty} P(n l ; s) \frac{r_{<}^{k}}{r_{>}^{k+1}} P\left(n^{\prime} l^{\prime} ; s\right) \mathrm{d} s
$$

where $r_{<}$and $r_{>}$are the lesser or greater of $r$ and $s$, respectively, and $P(n l ; r)$ and $P\left(n^{\prime} l^{\prime} ; r\right)$ are radial functions. If this function is known, then the Slater integral $R^{k}(a b, c d)$ is the simple one-dimensional integral

$$
R^{k}(a b, c d)=\int_{0}^{\infty} P(a ; r) P(c ; r) Y^{k}(b d ; r) \mathrm{d} r .
$$

When Jucys visited Hartree in Manchester in the summer of 1938, Hartree suggested that he investigate second-order differential equation methods and also apply the superposition of configuration ideas to carbon and its ions [3,4]. A year later, Jucys too published a paper with configuration interaction results for carbon that became the foundation of his thesis.

This period (1930-1940) defines the beginning of the computational atomic structure era. Calculations generally were done by hand. Hartree's student explored the application of Hartree's differential analyser to the solution of the Hartree equations without ex- 
change for chromium [5], but Hartree did not consider the accuracy sufficient [4]. Furthermore, the analyser was not suitable for equations with exchange.

Many changes have occurred since then that facilitate large scale computation. These changes were not only better methods for solving radial equations but also more powerful methods for evaluating expressions for matrix elements. During World War II, Jucys continued his research in the application of quantum mechanics to atomic structure theory. When he submitted his Doctoral thesis in 1952, one of his opponents drew his attention to the papers Racah [6] had published during the war. Jucys and his group immediately began to study Racah's theory [7]. Ultimately, it was in angular theory that Jucys made his greatest contribution.

The early 1960s was a period when the solution of the Hartree-Fock (HF) and multiconfiguration Hartree-Fock (MCHF) method using automatic electronic computers was the topic of research. By 1970, Hibbert's Weights program [8] was available for evaluating matrix elements as well as the first version of MCHF [9]. In 1988, the NJGRAF recoupling program by Bar-Shalom and Klapisch [10] based on Jucys diagrammatic method became available for speeding up the evaluation of matrix elements. In 1997, further improvements were made by Gaigalas et al. [11], through a code implementation that took advantage of several concepts - second quantization, quasi-spin, reduced coefficients of fractional parentage, and diagrammatic methods [12]. It provided a further speed-up of a factor of 2-6 depending on the case. But all these changes still treat the evaluation of the interaction matrix, element by element, though the NJGRAF implementation has a feature in which recoupling information can be reused. As a result, as wave function expansions grow in size, the files of data also grow. Files of the size of 10-20 gigabytes have been encountered. Fortunately, computer disks too have expanded so that the calculations are still possible, but cutting down on the angular data could avoid the necessity of the reading of large volumes of data and facilitate distributed computing on many processors. Current codes often degrade in performance to as low as a few percent of CPU usage (depending on the size of available memory) when heavy disk activity is needed for a large calculation. Thus, for large cases, extra computation will not reduce the total time.

In this paper, we propose a scheme that greatly reduces the data for large calculations, and eliminates much of the redundancy in present methods for angular computations. In fact, the data is largely independent of the size of a calculation, so that systematic methods, where orbital sets of increasing size are used, can readily be applied thereby facilitating the monitoring of convergence. It also means that, when a series of larger and larger calculations are performed, the angular integrations need not be repeated for each run. Our presentation will be for nonrelativistic LS wave function and the only operator will be the nonrelativistic Hamiltonian, $\mathbf{H}$, but the ideas can be generalized to $L S J$ (Breit-Pauli) or $j j$ (Dirac-Hartree-Fock) Hamiltonian and other operators.

\section{Systematic methods}

In an MCHF calculation, the wave function for an atomic state function (ASF) $|\psi(\gamma L S)\rangle$ is expanded in terms of a linear combination of configuration state functions (CSFs) $|(\gamma L S)\rangle$. Specifically,

$$
|\psi(\gamma L S)\rangle=\sum_{j}^{M} c_{j}\left|\left(\gamma_{j} L S\right)\right\rangle .
$$

Here $\gamma L S$ is a label for the atomic state whereas for the CSF it represents a configuration and its coupling, not necessarily only the final term. The CSFs are constructed from spin-orbitals as antisymmetrized linear combinations of products of spin-orbitals

$$
\phi_{n l m_{l} m_{s}}=\frac{1}{r} P_{n l}(r) Y_{l m_{l}}(\theta, \varphi) \xi_{m_{s}}(\sigma),
$$

where the radial functions $P_{n l}(r)$ are represented by their numerical values on a logarithmic grid, $Y_{l m_{l}}(\theta, \varphi)$ is a spherical harmonic, and $\xi_{m_{s}}(\sigma)$ a spin-function. The radial functions are required to be orthonormal within each $l$ symmetry. The multiconfiguration selfconsistent field (MC-SCF) procedure is used to optimize both the orbitals and the expansion coefficients to self-consistency.

In many calculations, orbitals can be divided into two sets: occupied and unoccupied. The latter are sometimes referred to as virtual or correlation orbitals. The occupied orbitals are present in the Hartree-Fock wave function or, more generally, in a zero-order approximation defined by a multireference set of CSFs. Wave function expansions can be generated by single $(\mathrm{S})$, double $(\mathrm{D})$, triple $(\mathrm{T})$, quadruple $(\mathrm{Q})$ excitations from the multireference set. In an excitation, an occupied orbital is replaced by an unoccupied, virtual orbital. In an active space method, any number of occupied orbitals may be replaced in which case a single reference configuration state is sufficient. 
The programs that generate expansions deal in terms of subshells of configurations, without regard to coupling $[13,14]$. For a given case, electrons are assigned to subshells. Electrons are then replaced by electrons from a list of allowed subshells according to some rule, and then all possible configuration states from the resulting configurations are generated. Thus the $2 s^{2} \rightarrow 3 p^{2}$ replacement, applied to the $1 s^{2} 2 s^{2} 2 p$ boron ground state configuration, generates all possible couplings (three in total) of the configuration $1 s^{2} 2 p 3 p^{2}$. Only one of these interacts with the CSF of the ground state. Restricting the expansion to only those CSFs that interact with one or more CSFs of the multireference set, in large cases may reduce the expansion by a factor of two or more with only a small effect on the energy.

The distinction between occupied and unoccupied applies readily to systems with filled shells, such as neon. Often CSFs of the multireference set contain unfilled subshells. For example, the ground state of boron is $1 s^{2} 2 s^{2} 2 p$ so one might say $1 s, 2 s, 2 p$ are occupied but the $2 p$ subshell is not filled. We certainly would like to replace $2 s^{2}$ by $2 p^{2}$ to get the $2 p^{3} \mathrm{CSF}$ but now we have excited two electrons from an occupied subshell to electrons in a partially filled subshell. For this reason, the orbitals of unfilled subshells are included in the virtual set, in practice. The replacement (or excitation) process is first done at the configuration level: one or more electrons in the initial configuration are replaced by electrons from the unfilled or unoccupied shells. Then antisymmetry rules are applied. Any distribution of electrons with an over-full subshell is rejected. Finally the angular momenta of the orbitals are coupled and only those replacements that yield a CSF of a given parity and term are accepted. Typically, the coupling of orbitals is from left to right, with the virtual orbitals that usually are assigned higher principal quantum numbers, coupled last.

One way of reducing an expansion, provided a firstorder calculation is sufficient, is to select only those CSFs that have a nonzero interaction matrix with one or more CSFs of the reference set. In large expansions, this may reduce the expansion size by as much as a half. A program LSREDUCE [15] has this function.

Angular calculations for the variational MCHF method, for example, perform angular integrations of the energy interaction matrix element by element. For an $M \times M$ matrix there are $M^{2}$ matrix elements (excluding symmetry) to be evaluated and for large calculations, particularly when LSREDUCE has not been used or when TQ excitations are present, many matrix elements are zero. Let $T_{\mathrm{z}}$ and $T_{\mathrm{nz}}$ be the time required to determine that a matrix element is zero and the average remaining time for an element that is nonzero, respectively. Let $f$ be the fraction of matrix elements that are nonzero. This fraction is often in the range of 0.01-0.1. Then the expected time for the angular integration for a calculation is

$$
T_{\text {total }}=M^{2}\left\{(1-f) T_{\mathrm{z}}+f T_{\mathrm{nz}}\right\} .
$$

From this equation it readily follows that, if

$$
T_{\mathrm{z}}>\frac{f}{1-f} T_{\mathrm{nz}},
$$

then more time will be spent on determining whether matrix elements are zero than for determining nonzero matrix elements. When $f=0.01$, the time required to detect a zero matrix element should be less than $T_{\mathrm{nz}} / 100$. In the past, the CPU time for angular calculations could be significantly reduced simply by checking that the two configurations did not differ by more than two electrons before any angular integrations were started. Clearly it is important to determine a zero matrix element as quickly as possible.

It should also be remembered that, whereas there are $M$ diagonal matrix elements, there are $M(M-1)$ nondiagonal ones. Expressed another way, the fraction of diagonal matrix elements is approximately $1 / M$ of all matrix elements. For $M$ in the range of $10^{5}-10^{6}$, this gets to be a vanishingly small fraction. Thus the efficiency of the calculation of the off-diagonal matrix elements is extremely important, more so than for the diagonal elements. Such calculations may require extended recoupling, recoupling that may be repeated frequently in the course of angular integrations for an expansion set. Grant and Quiney have some suggestions for how recoupling may be reduced to a minimum [16]. Another idea, first proposed by Weiss [17], was the use of a table look-up for interactions. The principal quantum numbers of the orbitals are not significant in angular calculations, except when electrons are equivalent: $1 s^{2} 2 s^{2} 2 p$ and $1 s^{2} 2 s 3 s\left({ }^{1} S\right) 2 p$ differ because in one case the $s$-electrons are equivalent whereas in the other they are not, but the matrix elements,

$$
\begin{array}{r}
\left\langle 1 s^{2} 2 s^{2} 2 p^{2} P|\mathbf{H}| 1 s^{2} 2 p^{3}{ }^{2} P\right\rangle, \\
\left\langle 1 s^{2} 3 s^{2} 2 p^{2} P|\mathbf{H}| 1 s^{2} 2 p^{3}{ }^{2} P\right\rangle, \\
\left\langle 1 s^{2} 2 s 3 s\left({ }^{1} S\right) 2 p^{2} P|\mathbf{H}| 1 s^{2} 2 p^{3}{ }^{2} P\right\rangle,
\end{array}
$$

are clearly related. All three have the same angular coupling, but the first two have equivalent electrons whereas the third does not and this affects the angular coefficient. More recently, a database idea has been 
suggested by Zatsarinny and Froese Fischer [18]. Assuming the table of database entries have been computed, both these methods replace the calculation of matrix elements by a search of a database for $M^{2}$ matrix elements or a fraction thereof, depending on the implementation and for large $M$ the time could be appreciable, particularly in an MCHF calculation where the angular data is accessed twice per iteration.

\section{Symbolic expansions}

In the discussions above, the angular integrations were always performed for a specific expansion without any assumptions about the order of the CSFs. The $M^{2}$ matrix elements were expressed in terms of radial integrals $I(n l)$ arising from the one-electron part of the Hamiltonian, and Slater integrals from the two-electron part. One change that has occurred since the early days of atomic structure calculations is that the angular integrations for the two-electron Coulomb matrix element between antisymmetrized states can readily be evaluated as required from the well-known formula:

$$
\begin{aligned}
& \left\langle\left(n_{1} l_{1}, n_{2} l_{2}\right) L S\left|\frac{1}{r_{12}}\right|\left(n_{3} l_{3}, n_{4} l_{4}\right) L^{\prime} S^{\prime}\right\rangle \\
& =\delta_{L S, L^{\prime} S^{\prime}} \mathcal{N} \sum_{k}\left[c_{k} R^{k}\left(n_{1} l_{1}, n_{2} l_{2} ; n_{3} l_{3}, n_{4} l_{4}\right)\right. \\
& \left.\quad+c_{k}^{\prime} R^{k}\left(n_{1} l_{1}, n_{2} l_{2} ; n_{4} l_{4}, n_{3} l_{3}\right)\right],
\end{aligned}
$$

where

$$
\begin{aligned}
c_{k}= & (-1)^{l_{1}+l_{3}-L}\left[l_{1}, l_{2}, l_{3}, l_{4}\right]^{1 / 2} \\
& \times\left(\begin{array}{ccc}
l_{1} & k & l_{3} \\
0 & 0 & 0
\end{array}\right)\left(\begin{array}{ccc}
l_{2} & k & l_{4} \\
0 & 0 & 0
\end{array}\right)\left\{\begin{array}{l}
l_{1} l_{2} L \\
l_{4} l_{3} k
\end{array}\right\}, \\
c_{k}^{\prime}= & (-1)^{l_{1}+l_{3}-S}\left[l_{1}, l_{2}, l_{4}, l_{3}\right]^{1 / 2} \\
& \times\left(\begin{array}{ccc}
l_{1} & k & l_{4} \\
0 & 0 & 0
\end{array}\right)\left(\begin{array}{ccc}
l_{2} & k & l_{3} \\
0 & 0 & 0
\end{array}\right)\left\{\begin{array}{l}
l_{1} l_{2} L \\
l_{3} l_{4} k
\end{array}\right\},
\end{aligned}
$$

and $\mathcal{N}$ is a renormalization factor:

$$
\mathcal{N}= \begin{cases}1 & \text { if } l_{1} \neq l_{2} \text { and } l_{3} \neq l_{4}, \\ \frac{1}{\sqrt{2}} & \text { if either } l_{1}=l_{2} \text { or } l_{3}=l_{4} \\ \frac{1}{2} & \text { but not both, } \\ \text { if } l_{1}=l_{2} \text { and } l_{3}=l_{4} .\end{cases}
$$

These angular integrations need not be repeated for the different values of $n_{1}, n_{2}, n_{3}, n_{4}$. Depending on the calculation, it may be that a table of expressions for such interactions may be useful, but this is an implementation decision.

With this in mind, let us express the ASF of a twoelectron system by a symbolic state that represents a linear combination of all possible two-electron configuration states, of given parity and $L S$, that can be constructed from a given orbital set, namely

$$
\left|\left(n l, n^{\prime} l^{\prime}\right) L S\right\rangle \equiv \sum_{n} \sum_{l} \sum_{n^{\prime}} \sum_{l^{\prime}}()\left|\left(n l, n^{\prime} l^{\prime}\right) L S\right\rangle .
$$

The expression on the left is symbolic and sufficient for angular integrations, where the four quantum numbers are treated as variables though triangle conditions will be needed to assure that coupling conditions are satisfied, and equivalent electrons need to be taken into account. The sum on the right-hand side is explicit. In practice, further conditions need to be considered when using the explicit form. Wave functions are expanded in terms of a basis of CSFs that are orthonormal. Thus those combinations of $\left(l, l^{\prime}\right)$ that are not allowed (whose CSFs are identically zero) must be eliminated and since CSFs are antisymmetric, $\left|\left(n l, n^{\prime} l\right) L S\right\rangle$ will not be orthogonal to $\left|\left(n^{\prime} l, n l\right) L S\right\rangle$. We define this as not part of the angular problem, but rather as part of the radial problem when symbolic states are used.

Let us express the ASF for the ${ }^{1} S$ ground state of helium by the approximation

$$
\psi\left(1 s^{2}{ }^{1} S\right) \approx\left|\left(n l, n^{\prime} l\right){ }^{1} S\right\rangle_{1 s}^{N L} .
$$

To indicate clearly the range of the orbital set, we have added the first and the last orbital to the symbolic state. The triangle condition now has required that $l=l^{\prime}$ and the orthonormality condition that $n^{\prime} \geq n$ so that the symbolic state on the right represents a sum

$$
\left|\left(n l, n^{\prime} l\right){ }^{1} S\right\rangle_{1 s}^{N L}=\sum_{n=1}^{N} \sum_{n^{\prime}=n}^{N} \sum_{0}^{L}()\left|\left(n l, n^{\prime} l\right){ }^{1} S\right\rangle .
$$

Then no angular data would be needed for the evaluation of the matrix elements since all can be derived from formulas. Notice that Eq. (13) represents $N(N+1) / 2 \times(L+1)$ CSFs. "Systematic" calculations are ones where the orbital basis is systematically increased so that convergence of an atomic property can be monitored.

Similarly,

$$
\psi\left(1 s 2 p^{1,3} P^{\circ}\right) \approx\left|\left(n l, n^{\prime}(l+1)\right)^{1,3} P^{\circ}\right\rangle_{1 s}^{N L},
$$


where the explicit form of the symbolic state is defined as

$$
\begin{aligned}
& \left|\left(n l, n^{\prime} l^{\prime}\right)^{1,3} P^{\circ}\right\rangle_{1 s}^{N L} \\
& \quad=\sum_{n=1}^{N} \sum_{l=0}^{L} \sum_{n^{\prime}=2}^{N} \sum_{l^{\prime}=l+1}^{L}()\left|\left(n l, n^{\prime} l^{\prime}\right)^{1,3} P^{\circ}\right\rangle .
\end{aligned}
$$

Expansions such as the above were used to study the calculation of two-electron wave functions using a B-spline basis [19]. In a configuration interaction (CI) calculation, the radial functions are assumed to be known, and only the eigenvalue and eigenvector of an interaction matrix are to be determined. Given that the calculation of the eigenvalue and eigenvector by the Davidson method [20] depends solely on matrix-vector multiplication, one could anticipate evaluating a part of the matrix "on the fly", immediately multiplying by the appropriate components of the vector, and never storing the matrix itself.

Since the Hamiltonian for a many-electron system is always a two-electron operator, CSFs that differ by more than two electrons will not interact. Thus single (S) and double (D) excitations play a very special role in atomic structure calculations. For simple structures, such as $1 s^{2} 2 s^{2} 2 p^{6} n l$ of $\mathrm{Na}$, good results can be obtained by such a process. But the situation can be considerably more complex. For example, for the even $3 s 3 p^{4}{ }^{2} P$ term in S II [21], there is strong mixing of $3 s 3 p^{4}{ }^{2} P, 3 s^{2} 3 p^{2}\left({ }^{3} P\right) 4 d^{2} P, 3 s^{2} 3 p^{2}\left({ }^{1} D\right) 3 d^{2} P$ configuration states, and the strong $3 s^{2} \rightarrow 3 p^{2}$ interaction also needs to be included in a good zero-order wave function. In order to get the levels of this spectrum correct with an orthogonal orbital basis, the following reference set was needed:

$$
\begin{aligned}
& \left\{3 s 3 p^{4}, 3 s 3 p^{3} 4 p, 3 s^{2} 3 p^{2} 3 d,\right. \\
& \left.\quad 3 s^{2} 3 p^{2} 4 d, 3 p^{4} 3 d, 3 p^{4} 4 d\right\}^{2} P .
\end{aligned}
$$

All possible couplings were included.

Though the general case may be extremely complex, let us first consider a simple case, such as the $1 s^{2} 2 s^{2} 2 p^{6}$ ground state of neon where subshells are either filled or unoccupied. Then the wave function from SD excitations may be expressed as

$$
\psi=\Psi_{\mathrm{ref}}+\Psi_{\mathrm{S}}+\Psi_{\mathrm{D}} .
$$

The symbolic states for $\psi_{\mathrm{S}}$ are formed by removing an occupied electron and replacing it by one from the virtual shells. From a coupling point of view, this means we have a coupled subconfiguration $\left(\Psi_{\mathrm{sub}_{c}}\right) L_{c} S_{c}$ and a virtual orbital $v l$. Then we get

$$
\Psi_{\mathrm{S}}=\sum_{c}()\left|\left\{\left(\Psi_{\mathrm{sub}_{c}}\right) L_{c} S_{c},(v l)^{2} L\right\} L S\right\rangle .
$$

In the case of neon, there are three symbolic states that define $\Psi_{\mathrm{S}}$, namely:

1. $\left|\left\{\Psi\left(1 s^{2} 2 s^{2} 2 p^{5}\right)^{2} P,(v p)^{2} P\right\}{ }^{1} S\right\rangle$.

2. $\left|\left\{\Psi\left(1 s^{2} 2 s 2 p^{6}\right)^{2} S,(v s)^{2} S\right\}{ }^{1} S\right\rangle$.

3. $\left|\left\{\Psi\left(1 s 2 s^{2} 2 p^{6}\right)^{2} S,(v s)^{2} S\right\}{ }^{1} S\right\rangle$.

One of the properties of a Hartree-Fock approximation is that, in this case, the matrix element $\left\langle\Psi_{\mathrm{ref}}|\mathbf{H}| \Psi_{S}\right\rangle=0$, but when the same functions are used in a Breit-Pauli calculation this no longer holds since the Hamiltonian has changed.

In the case of two excitations, two electrons are "pulled out" and replaced by electrons from unoccupied shells or, in terms of coupled configuration states, two orbitals are uncoupled and replaced by virtual orbitals. Rather than couple the resulting configuration from left to right, we first couple the two virtual orbitals to a resultant coupling and then couple the two components from left to right. Hence

$$
\Psi_{\mathrm{D}}=\sum_{c}\left|\left\{\left(\Psi_{\mathrm{sub}_{c}}\right) L_{c} S_{c},\left(v_{1} l_{1}, v_{2} l_{2}\right) L_{v} S_{v}\right\} L S\right\rangle,
$$

where for emphasis we use the notation $v l$ rather than $n l$ to designate a virtual orbital. In the case of neon there are now eleven (11) symbolic states, namely:

1. $\left|\left\{\Psi\left(1 s^{2} 2 s^{2} 2 p^{4}\right)^{3} P,\left(v_{1} l_{1}, v_{2} l_{2}\right){ }^{3} P\right\}{ }^{1} S\right\rangle$.

2. $\left|\left\{\Psi\left(1 s^{2} 2 s^{2} 2 p^{4}\right)^{1} D,\left(v_{1} l_{1}, v_{2} l_{2}\right){ }^{1} D\right\}{ }^{1} S\right\rangle$.

3. $\left|\left\{\Psi\left(1 s^{2} 2 s^{2} 2 p^{4}\right)^{1} S,\left(v_{1} l_{1}, v_{2} l_{2}\right)^{1} S\right\}^{1} S\right\rangle$.

4. $\left|\left\{\Psi\left(1 s^{2} 2 s 2 p^{5}\right)^{1} P,\left(v_{1} l_{1}, v_{2} l_{2}\right)^{1} P\right\}{ }^{1} S\right\rangle$.

5. $\left|\left\{\Psi\left(1 s^{2} 2 s 2 p^{5}\right)^{3} P,\left(v_{1} l_{1}, v_{2} l_{2}\right)^{3} P\right\}{ }^{1} S\right\rangle$.

6. $\left|\left\{\Psi\left(1 s^{2} 2 p^{6}\right)^{1} S,\left(v_{1} l_{1}, v_{2} l_{2}\right){ }^{1} S\right\}{ }^{1} S\right\rangle$.

7. $\left|\left\{\Psi\left(1 s 2 s^{2} 2 p^{5}\right)^{1} P,\left(v_{1} l_{1}, v_{2} l_{2}\right)^{1} P\right\}{ }^{1} S\right\rangle$.

8. $\left|\left\{\Psi\left(1 s 2 s^{2} 2 p^{5}\right)^{3} P,\left(v_{1} l_{1}, v_{2} l_{2}\right)^{3} P\right\}^{1} S\right\rangle$.

9. $\left|\left\{\Psi\left(1 s 2 s 2 p^{6}\right)^{1} S,\left(v_{1} l_{1}, v_{2} l_{2}\right){ }^{1} S\right\}{ }^{1} S\right\rangle$.

10. $\left|\left\{\Psi\left(1 s 2 s 2 p^{6}\right)^{3} S,\left(v_{1} l_{1}, v_{2} l_{2}\right)^{3} S\right\}{ }^{1} S\right\rangle$.

11. $\left|\left\{\Psi\left(2 s^{2} 2 p^{6}\right){ }^{1} S,\left(v_{1} l_{1}, v_{2} l_{2}\right){ }^{1} S\right\}{ }^{1} S\right\rangle$.

This form of coupling where the coupling of the virtual orbitals is derived from the coupling of a configuration state in the reference set was called "parent-coupling" by Weiss [22] who claimed that it could reduce the expansion by as much as a factor of three. In the above list, the first six symbolic states are a part of the valence correlation, 7-10 are a part of the core-valence correlation, and the last one is the core correlation, assuming that the core is defined to be $1 s^{2}$. 


\section{Coulomb matrix elements between symbolic states}

Let us now generalize the above examples to $N$-electron systems and consider expressions for Coulomb matrix elements between states where one or both may be symbolic states.

Symbolic states are generated from single and double excitations from members of the reference set. Let us first consider double excitations. Let $\left|\Psi_{\text {ref }}\right\rangle$ be an arbitrary $L S$-coupled antisymmetric configuration state function for $N$-electrons. A doubly excited symbolic state $|\Psi\rangle$ is formed by uncoupling a pair of electrons, leaving an $(N-2)$ subconfiguration, replacing the "pulled out" electrons, say $\left(n_{c 1} l_{c 1}, n_{c 2} l_{c 2}\right)$, by coupled virtual orbitals $\left(v_{1} l_{1}, v_{2} l_{2}\right) L_{v} S_{v}$ so that

$$
|\Psi\rangle=\left|\left\{\left(\Psi_{\mathrm{sub}}\right) L_{c} S_{c},\left(v_{1} l_{1}, v_{2} l_{2}\right) L_{v} S_{v}\right\} L S\right\rangle .
$$

For brevity, we may refer to $\left|\left(v_{1} l_{1}, v_{2} l_{2}\right) L_{v} S_{v}\right\rangle$ as $\left|\left(\psi_{v}\right) L_{v} S_{v}\right\rangle$ or simply as $\left|\left(l_{1}, l_{2}\right) L_{v} S_{v}\right\rangle$ when only angular quantum numbers are relevant. By allowing the virtual pairs $\left(v_{1} l_{1}, v_{2} l_{2}\right)$ to range over all possible pairs, we can view these quantities as variables and $\left|\left\{\left(\Psi_{\mathrm{sub}}\right) L_{c} S_{c},\left(v_{1} l_{1}, v_{2} l_{2}\right) L_{v} S_{v}\right\} L S\right\rangle$ as a symbolic state. In subsequent expressions the principal quantum numbers will be omitted in symbolic states. It may happen that the same symbolic state can be generated from two different members of the reference set, but every doubly excited symbolic state will differ by two electrons from every member of the reference set.

Let us define the $N$-electron Coulomb operator to be $\mathbf{G}$ and consider the interaction between any member of the reference set and a doubly excited symbolic state. Let $\left(n_{c 1} l_{c 1}, n_{c 2} l_{c 2}\right)$ be the two electrons in the reference configuration state that differ from those in the symbolic state. Define

$$
\left|\Psi^{\prime \prime}\right\rangle=\left|\left\{\left(\Psi_{\mathrm{sub}}\right) L_{c} S_{c},\left(n_{c 1} l_{c 1}, n_{c 2} l_{c 2}\right) L_{v} S_{v}\right\} L S\right\rangle .
$$

Then

$$
\begin{aligned}
& \left\langle\Psi|\mathbf{G}| \Psi_{\text {ref }}\right\rangle \\
& \quad=\left\langle\left(l_{1}, l_{2}\right) L_{v} S_{v}|\mathbf{G}|\left(l_{c 1}, l_{c 2}\right) L_{v} S_{v}\right\rangle\left\langle\Psi^{\prime \prime} \mid \Psi_{\text {ref }}\right\rangle .
\end{aligned}
$$

This result assumes that the one-electron orbitals are orthogonal and that the virtual orbitals are orthogonal to the occupied orbitals. For this matrix element to be nonzero the triangle condition $\left(l_{c 1} l_{c 2} L_{v}\right)$ needs to be satisfied. Limiting the $L_{v}$ for virtual orbitals to the angular quantum number allowed for occupied orbitals is what leads to "parent" coupling. The interaction may also be zero if the overlap $\left\langle\Psi^{\prime \prime} \mid \Psi_{\text {ref }}\right\rangle$ is zero. However, in practice, there should be at least one member of the reference set for which this overlap is nonzero.

The matrix element between two symbolic states $\left\langle\Psi^{\prime}|\mathbf{G}| \Psi\right\rangle$ can be partitioned into three parts:

$$
\left\langle\Psi^{\prime}|\mathbf{G}| \Psi\right\rangle=\mathcal{M}_{v v}+\mathcal{M}_{c v}+\mathcal{M}_{c c} .
$$

Here

$$
\begin{aligned}
\mathcal{M}_{v v}= & \left\langle\left(\Psi_{\mathrm{sub}}^{\prime}\right) L_{c}^{\prime} S_{c}^{\prime} \mid\left(\Psi_{\mathrm{sub}}\right) L_{c} S_{c}\right\rangle \\
& \times\left\langle\left(\psi_{v}^{\prime}\right) L_{v}^{\prime} S_{v}^{\prime}|\mathbf{G}|\left(\psi_{v}\right) L_{v} S_{v}\right\rangle \\
\mathcal{M}_{c c}= & \left\langle\left(\Psi_{\mathrm{sub}}^{\prime}\right) L_{c}^{\prime} S_{c}^{\prime}|\mathbf{G}|\left(\Psi_{\mathrm{sub}}\right) L_{c} S_{c}\right\rangle \\
& \times\left\langle\left(\psi_{v}^{\prime}\right) L_{v}^{\prime} S_{v}^{\prime} \mid\left(\psi_{v}\right) L_{v} S_{v}\right\rangle, \\
\mathcal{M}_{c v}= & \sum_{l_{c}^{\prime}, l_{c}} \sum_{l_{v}^{\prime}, l_{v}} \sum_{L_{G}, S_{G}} \mathcal{C}_{\mathrm{sym}} \\
& \times\left\langle\left(l_{c}^{\prime}, l_{v}^{\prime}\right) L_{G} S_{G}|\mathbf{G}|\left(l_{c}, l_{v}\right) L_{G} S_{G}\right\rangle .
\end{aligned}
$$

Notice the overlap integral in the definition of $\mathcal{M}_{v v}$ and also $\mathcal{M}_{c c}$. There will be no contribution from the former if the subconfigurations or their coupling are different whereas for the latter there will be no contribution if the virtual pairs are different.

Contributions from $\mathcal{M}_{c v}$ arise when a virtual orbital from the left symbolic state is the same as a virtual orbital on the right. This is the contribution where the calculation of the matrix element requires extensive recoupling and the two-electron interaction involves an orbital from the subconfiguration. In Eq. (26), $l_{c}, l_{c}^{\prime}$ run over all subconfiguration orbitals, and $l_{v}, l_{v}^{\prime}$ over the two correlation orbitals of the left and right symbolic configuration states, respectively. The values of $L_{G}, S_{G}$ are limited by triangle relations $\left(l_{v} l_{c} L_{G}\right)$, $\left(l_{v}^{\prime} l_{c}^{\prime} L_{G}\right)$, and $\left(\frac{1}{2} \frac{1}{2} S_{G}\right)$. The coefficient $\mathcal{C}_{\text {sym }}$ is expressed [23] as

$$
\begin{aligned}
& \mathcal{C}_{\text {sym }} \\
& \left.\left.=2\left\langle\overline{l_{v}^{\prime}} \mid \overline{l_{v}}\right\rangle\left(\overline{l_{v}}, l_{v} \mid\right\}\left(l_{1} l_{2}\right) L_{v} S_{v}\right)\left(\overline{l_{v}^{\prime}}, l_{v}^{\prime} \mid\right\}\left(l_{1}^{\prime} l_{2}^{\prime}\right) L_{v}^{\prime} S_{v}^{\prime}\right) \\
& \times \sum_{k, \kappa} \Gamma_{k \kappa}\left(\Psi_{\mathrm{sub}}^{\prime}, l_{c}^{\prime} ; \Psi_{\mathrm{sub}}, l_{c}\right)\left[L_{G}, S_{G}, k, \kappa\right] \\
& \times\left[L_{v}, S_{v}, L_{v}^{\prime}, S_{v}^{\prime}\right]^{1 / 2} \\
& \times(-1)^{L_{c}+L+L_{G}+l_{v}^{\prime}-l_{v}+\overline{l_{v}}-l_{c}+k}(-1)^{S_{c}+S+S_{G}+\kappa} \\
& \times\left\{\begin{array}{lll}
L_{v} & k & L_{v}^{\prime} \\
L_{c}^{\prime} & L & L_{c}
\end{array}\right\}\left\{\begin{array}{lll}
L_{v} & k & L_{v}^{\prime} \\
l_{v}^{\prime} & l_{v} & l_{v}
\end{array}\right\}\left\{\begin{array}{lll}
l_{c} & k & l_{c}^{\prime} \\
l_{v}^{\prime} & L_{G} & l_{v}
\end{array}\right\} \\
& \times\left\{\begin{array}{c}
S_{v} \kappa S_{v}^{\prime} \\
S_{c}^{\prime} S S_{c}
\end{array}\right\}\left\{\begin{array}{ccc}
S_{v} & \kappa & S_{v}^{\prime} \\
\frac{1}{2} & \frac{1}{2} & \frac{1}{2}
\end{array}\right\}\left\{\begin{array}{lll}
\frac{1}{2} & \kappa & \frac{1}{2} \\
\frac{1}{2} & S_{G} & \frac{1}{2}
\end{array}\right\} .
\end{aligned}
$$


In this equation, $\left\langle\overline{l_{v}} \mid \overline{l_{v}^{\prime}}\right\rangle$ is the overlap between the virtual orbitals not involved in the interaction and $\left.\left(\overline{l_{v}}, l_{v} \mid\right\}\left(l_{1} l_{2}\right) L_{v} S_{v}\right)$ is the two-electron mixed-shell coefficient of fractional parentage:

$$
\begin{aligned}
& \left.\left(\overline{l_{v}}, l_{v} \mid\right\}\left(l_{1} l_{2}\right) L_{v} S_{v}\right) \\
& =\left\{\begin{array}{l}
\frac{1}{2}\left[1+(-1)^{L_{v}+S_{v}}\right] \\
\text { if } l_{1}, l_{2} \text { are equivalent, } \\
\frac{1}{\sqrt{2}}\left\{\delta_{l_{v} l_{2}}+\delta_{l_{v} l_{1}}(-1)^{l_{1}+l_{2}+L_{v}+S_{v}}\right\} \\
\text { otherwise. }
\end{array}\right.
\end{aligned}
$$

The quantity $\Gamma_{k \kappa}\left(\Psi_{\text {sub }}^{\prime}, l_{c}^{\prime} ; \Psi_{\text {sub }}, l_{c}\right)$ depends only on the internal quantum numbers of the subconfigurations and not on the virtual orbitals. It is conveniently expressed as the coefficient in the expansion of an $(N-2)$-electron matrix element of a general onebody double-tensor operator,

$$
W_{q \tau}^{[k \kappa]}=\sum_{i=1}^{N-2} w_{q \tau}^{[k \kappa]}(i),
$$

in terms of one-electron matrix elements:

$$
\begin{aligned}
& \left\langle\Psi_{\mathrm{sub}}^{\prime} L_{c}^{\prime} S_{c}^{\prime}\left\|W^{[k \kappa]}\right\| \Psi_{\mathrm{sub}} L_{c} S_{c}\right\rangle \\
& \quad=\sum_{l_{c}^{\prime}, l_{c}} \Gamma_{k \kappa}\left(\Psi_{\mathrm{sub}}^{\prime}, l_{c}^{\prime} ; \Psi_{\mathrm{sub}}, l_{c}\right)\left\langle l_{c}^{\prime}\left\|w^{[k \kappa]}\right\| l_{c}\right\rangle .
\end{aligned}
$$

Here, $k$ is the tensor rank of the operator with respect to the orbital angular momentum, and $\kappa$ is the tensor rank with respect to the spin angular momentum. The possible values of $k$ and $\kappa$ in the sum of Eq. (23) are limited by triangle relations $\left(l_{c}^{\prime} k l_{c}\right)$ and $\left(\frac{1}{2} \kappa \frac{1}{2}\right)$, so that $\kappa$ is either 0 (singlet) or 1 (triplet).

Thus, in general the coefficient $\mathcal{C}_{\text {sym }}$ is a sum of products of several factors: an overlap integral; factors that depend on orbital quantum numbers and would need to be evaluated at run time when the quantum numbers of the virtual orbitals are known; and other factors, including the quantities $\Gamma_{k \kappa}\left(\Psi_{\mathrm{sub}}^{\prime}, l_{c}^{\prime} ; \Psi_{\mathrm{sub}}, l_{c}\right)$, that could be evaluated as part of the angular integration process and treated as a set of constants for each interacting pair of symbolic states.

Similar expressions can be derived for interactions with symbolic states formed from single substitutions.

Thus, all the matrix elements between the reference and symbolic states ( $\mathrm{S}$ or $\mathrm{D}$ ) and between two symbolic states can be expressed as a sum of terms, where each term is a product of three factors:
(1) a quantity independent of the virtual orbitals,

(2) a quantity dependent on the angular quantum numbers of the virtual orbitals and whether they are equivalent or nonequivalent,

(3) a two-electron matrix element.

Note that the first two quantities involve no radial functions, while the third includes both angular and radial integrals. This factorization is seen clearly in the equations above for $\mathcal{M}_{v v}$ and $\mathcal{M}_{c c}$, but the situation for $\mathcal{M}_{c v}$ is more complicated. To see this case more explicitly, we can write

$$
\mathcal{C}_{\text {sym }}=\sum_{k} \mathcal{D}_{k} \mathcal{A}_{k}
$$

with

$$
\begin{aligned}
\mathcal{D}_{k}= & \sum_{\kappa} \Gamma_{k \kappa}\left(\Psi_{\mathrm{sub}}^{\prime}, l_{c}^{\prime} ; \Psi_{\mathrm{sub}}, l_{c}\right) \\
& \times\left[S_{G}, k, \kappa\right]\left[L_{v}, S_{v}, L_{v}^{\prime}, S_{v}^{\prime}\right]^{1 / 2} \\
& \times(-1)^{L_{c}+L-l_{c}+k}(-1)^{S_{c}+S+S_{G}+\kappa} \\
& \times\left\{\begin{array}{lll}
L_{v} & k & L_{v}^{\prime} \\
L_{c}^{\prime} & L & L_{c}
\end{array}\right\}\left\{\begin{array}{lll}
S_{v} & \kappa & S_{v}^{\prime} \\
S_{c}^{\prime} & S & S_{c}
\end{array}\right\} \\
& \times\left\{\begin{array}{ccc}
S_{v} & \kappa & S_{v}^{\prime} \\
\frac{1}{2} & \frac{1}{2} & \frac{1}{2}
\end{array}\right\}\left\{\begin{array}{lll}
\frac{1}{2} & \kappa & \frac{1}{2} \\
\frac{1}{2} & S_{G} & \frac{1}{2}
\end{array}\right\},
\end{aligned}
$$

and

$$
\begin{aligned}
\mathcal{A}_{k}= & \left.\left.2\left(\overline{l_{v}}, l_{v} \mid\right\}\left(l_{1} l_{2}\right) L_{v} S_{v}\right)\left(\overline{l_{v}^{\prime}}, l_{v}^{\prime} \mid\right\}\left(l_{1}^{\prime} l_{2}^{\prime}\right) L_{v}^{\prime} S_{v}^{\prime}\right) \\
& \times\left\langle\overline{l_{v}^{\prime}} \mid \overline{l_{v}}\right\rangle\left[L_{G}\right](-1)^{\overline{l_{v}}}+L_{G}+l_{v}^{\prime}-l_{v} \\
& \times\left\{\begin{array}{ccc}
L_{v} & k & L_{v}^{\prime} \\
l_{v}^{\prime} & \bar{l}_{v} & l_{v}
\end{array}\right\}\left\{\begin{array}{ccc}
l_{c} & k & l_{c}^{\prime} \\
l_{v}^{\prime} & L_{G} & l_{v}
\end{array}\right\} .
\end{aligned}
$$

Now we see that the set of coefficients $\mathcal{A}_{k}$ must be computed as part of the "radial" calculation for each choice of virtual orbitals, while the set of coefficients $\mathcal{D}_{k}$ can be computed at the beginning as part of the "angular" segment of the problem.

These results have been derived [23] under the assumption that the virtual (or correlation) orbitals were distinct from the occupied orbitals. The extent to which this separation of occupied and virtual orbitals is necessary requires further investigation. Clearly the subconfigurations consist only of occupied orbitals.

Of course, in a subsequent radial calculation, a symbolic state function will need to be expanded into the usual CSFs, by allowing the virtual orbitals to run over 
the set of all possible values and the required coupling. This defines a block of CSFs, all with similar angular properties. The angular data now is an expression for the interaction of one block either with itself or with another block: i. e. the interaction of one set of CSFs with another. This has imposed an order on the CSFs but with this order, the angular information is independent of the size of the virtual orbital set. The basic element for the evaluation of an interaction matrix is the two-electron matrix element. Whereas in existing codes, a calculation starts with the evaluation of all possible Slater integrals that can be derived from a set of orbitals, in the present scheme two-electron matrix elements will be needed. Many possibilities exist as to how this can be implemented.

\section{A symbolic expansion for $1 s^{2} 2 s^{2}{ }^{1} S$ of beryllium}

Let us now consider the beryllium ground state with a two-configuration reference set, namely

$$
\left\{1 s^{2} 2 s^{2}, 1 s^{2} 2 p^{2}\right\}^{1} S .
$$

Then the zero-order wave function is

$$
\Psi_{0}=c_{1}\left|1 s^{2} 2 s^{2}{ }^{1} S\right\rangle+c_{2}\left|1 s^{2} 2 p^{2}{ }^{1} S\right\rangle .
$$

For simplicity, let us consider only symbolic states for double excitations and omitting excitations from $1 s^{2}$.

Then we get the following symbolic states:

1. $\left|\left\{(1 s, 1 s){ }^{1} S,\left(v_{1} l_{1}, v_{2} l_{2}\right){ }^{1} S\right\}{ }^{1} S\right\rangle$.

2. $\left|\left\{(1 s, 2 s){ }^{1} S,\left(v_{1} l_{1}, v_{2} l_{2}\right){ }^{1} S\right\}{ }^{1} S\right\rangle$.

3. $\left|\left\{(1 s, 2 s){ }^{3} S,\left(v_{1} l_{1}, v_{2} l_{2}\right){ }^{3} S\right\}{ }^{1} S\right\rangle$.

4. $\left|\left\{(1 s, 2 p){ }^{1} P,\left(v_{1} l_{1}, v_{2} l_{2}\right){ }^{1} P\right\}{ }^{1} S\right\rangle$.

5. $\left|\left\{(1 s, 2 p){ }^{3} P,\left(v_{1} l_{1}, v_{2} l_{2}\right){ }^{3} P\right\}{ }^{1} S\right\rangle$.

Note that the first symbolic state could be derived from either member of the reference set. It is part of "valence correlation" whereas the remaining symbolic states are part of core-valence correlation. Not included are contributions from the core-core correlation.

Appendix A shows the angular coefficient file for the beryllium example. The numerical coefficients are obtained by evaluating all the quantities in Eqs. (23)(27) that are independent of the quantum numbers of the correlation orbitals. $L S$ coupling has been used throughout.

The data includes the minimum information needed to derive the expression for a matrix element. Terms that depend on $A(k)$ do not include the sum over the two possible orderings on the left and the two possible orders on the right. For antisymmetric functions, it needs to be remembered that

$$
\begin{aligned}
& \left|\left(n_{1} l_{1}, n_{2} l_{2}\right) L S\right\rangle \\
& \quad=-(-1)^{l_{1}+l_{2}-L+1 / 2+1 / 2-S}\left|\left(n_{2} l_{2}, n_{1} l_{1}\right) L S\right\rangle
\end{aligned}
$$

or

$$
\begin{aligned}
& \left|\left(n_{1} l_{1}, n_{2} l_{2}\right) L S\right\rangle \\
& \quad=(-1)^{l_{1}+l_{2}-L+2-S}\left|\left(n_{2} l_{2}, n_{1} l_{1}\right) L S\right\rangle .
\end{aligned}
$$

Thus the contribution

$\langle(1 \mathrm{~s}, \mathrm{v} 1) 3 \mathrm{~L}|\mathrm{G}| \quad(1 \mathrm{~s}, \mathrm{v} 3) 3 \mathrm{~L}\rangle\langle\mathrm{V} 2 \mid \mathrm{V} 4\rangle$

found in Appendix A must be expanded (in the same notation) to

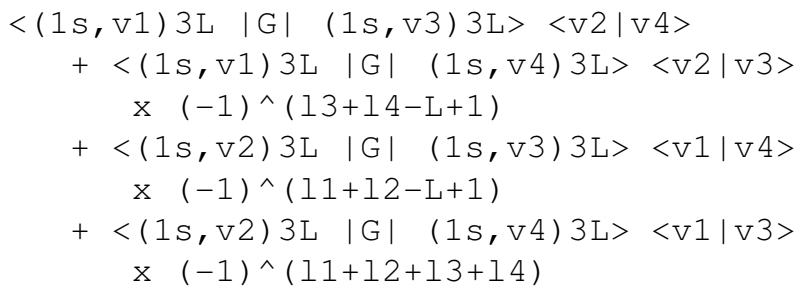

In Appendix B we show the expressions for interactions within the first symbolic state (representing valence correlation) when orbitals are restricted to $s^{-}, p-$, or $d$-orbitals. Notice now the extensive enumeration of cases and the increase of data. All CSFs associated with this symbolic state interact, and so this block of the interaction matrix is dense. In general, at this level of expansion of the energy expressions, the sparsity structure of the interaction matrix can be evaluated.

Figure 1 shows the structure of the interaction matrix between the symbolic states when the virtual orbitals are from the set $n l$ with $n$ in the range from 3 to 8 , and $l \leq 3$. Not included in this figure is the contribution from single substitutions nor the interaction with the reference set. The number of nonzero matrix elements is $33.3 \%$ and all interact with one or more of the elements of the reference set. In the present scheme, by coupling a subconfiguration with a two-electron state, the zero matrix elements can readily be identified from overlap integrals and selection rules.

\section{Conclusion}

Existing large scale atomic structure calculations produce many gigabytes of angular data, that can reduce CPU performance dramatically, to a few percent 


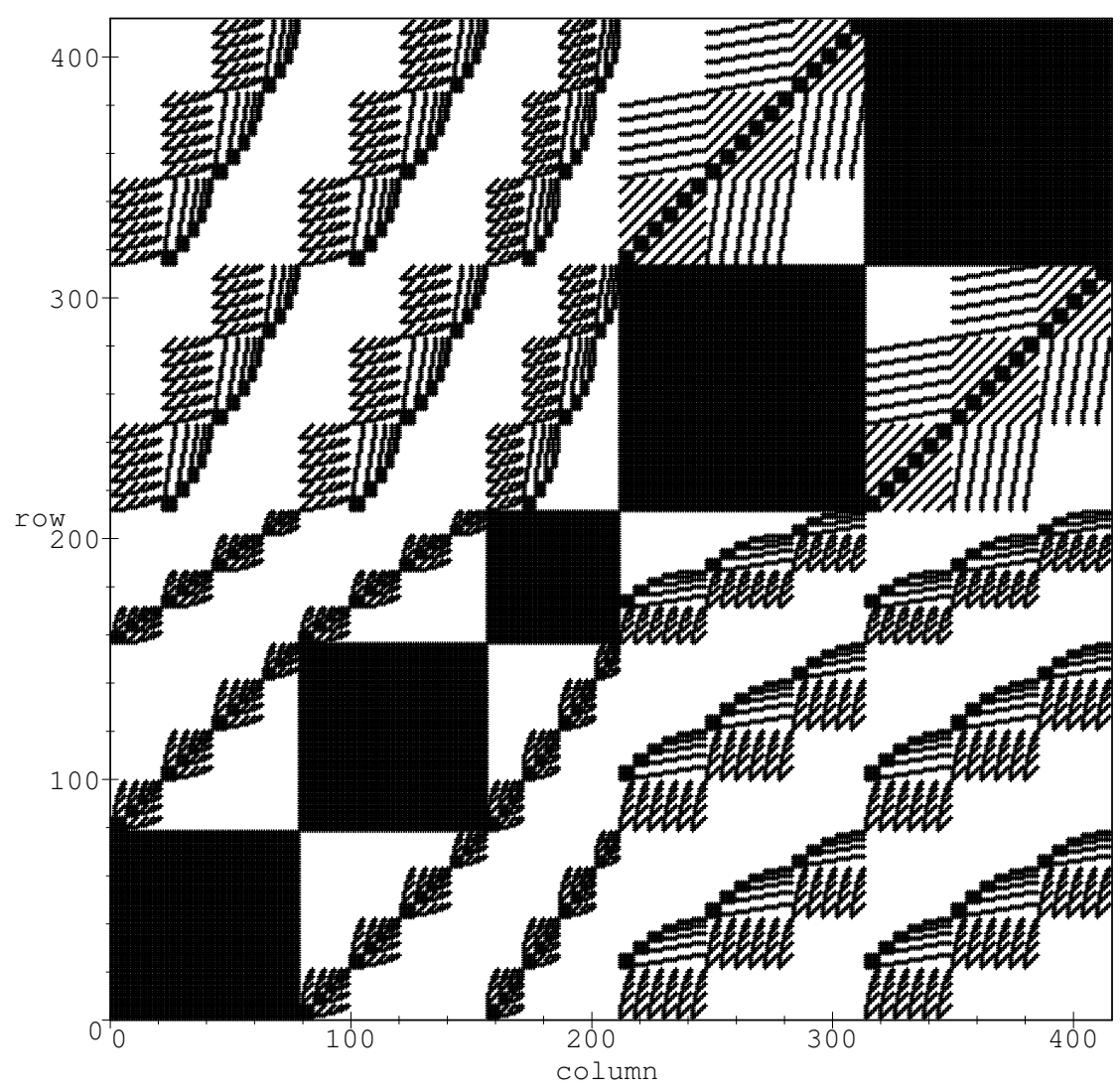

Fig. 1. Structure of the interaction between the valence and core-polarization symbolic states in the Be ground state with the multireference set of $\left\{1 s^{2} 2 s^{2}, 1 s^{2} 2 p^{2}\right\}{ }^{1} S$. (Courtesy of R. Matulioniené [23].)

if not enough memory is available and in extreme cases, the calculation may simply be impossible. By expressing a wave function in terms of an expansion of symbolic states consisting of two components - a subconfiguration and a coupled pair of virtuals - the angular data is greatly reduced without affecting the accuracy of the wave function. A further reduction is achieved by expressing the interaction in terms of interactions between two-electron configuration states without the necessity of knowing the angular quantum numbers of the interacting electrons. But most important is the fact that the data is independent of the orbital set. During an application of this approach, the symbolic states in an expansions will need to be expanded into a block of configuration states. The size of this block will depend on the size of the orbital set. The angular data for an interaction between two symbolic states is sufficient to generate all the interactions between the two blocks of configuration states. It is conceivable that large calculations can be performed in memory without the need for large volumes of data on disk.

The new scheme requires a reformulation of the angular integrations, involving recouplings and fractional parentage in different ways. Derivations of these new expressions were greatly facilitated by Jucys' diagrammatic methods [24].

\section{Appendix A. Angular coefficients for the Be $1 s^{2} 2 s^{2}{ }^{1} S$ example: minimum data}

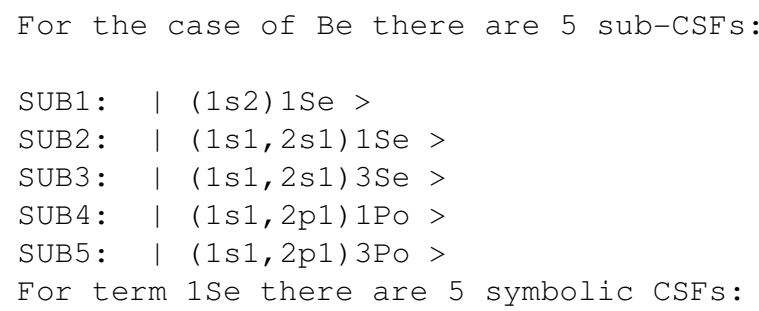




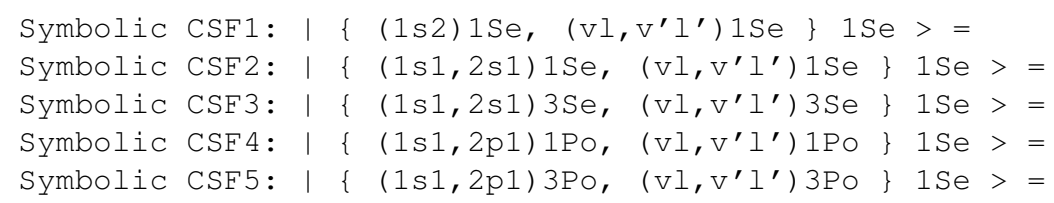

INTERACTION BLOCKS FOR G CONSERVING L, S

Block $(1 \mathrm{X} 1):<\{(\mathrm{Sub} 1) 1 \mathrm{Se},(\mathrm{v} 1, \mathrm{v} 2) 1 \mathrm{Se}\} 1 \mathrm{Se}|\mathrm{G}|\{(\mathrm{Sub} 1) 1 \mathrm{Se},(\mathrm{v} 3, \mathrm{~V} 4) 1 \mathrm{Se}\} 1 \mathrm{Se}>=$ $<(\mathrm{v} 1, \mathrm{v} 2) 1 \mathrm{Se}|\mathrm{G}|(\mathrm{v} 3, \mathrm{v} 4) 1 \mathrm{Se}>+<(\mathrm{Sub} 1) 1 \mathrm{Se} \mid$ (Sub1) 1Se $>+$

$<(\mathrm{Sub} 1) 1 \mathrm{Se}|\mathrm{G}|(\mathrm{Sub} 1) 1 \mathrm{Se}>\langle(\mathrm{v} 1, \mathrm{v} 2) 1 \mathrm{Se} \mid(\mathrm{v} 3, \mathrm{v} 4) 1 \mathrm{Se}\rangle \quad+$

$1 / 2 \mathrm{~A}(\mathrm{k}=0) \quad\langle(1 \mathrm{~s}, \mathrm{~V} 1) 1 \mathrm{~L}|\mathrm{G}| \quad(1 \mathrm{~s}, \mathrm{~V} 3) 1 \mathrm{~L}\rangle \quad\langle\mathrm{V} 2 \mid \mathrm{V} 4\rangle+$

$3 / 2 A(k=0) \quad\langle(1 s, v 1) 3 L \quad|G| \quad(1 s, v 3) 3 L\rangle \quad\langle v 2 \mid v 4\rangle$

Block (2X1) : < \{(Sub2)1Se, (v1,v2)1Se\}1Se | G | \{(Sub1) 1Se, (v3,v4) 1Se $\} 1 \mathrm{Se}>=$ $<(\mathrm{v} 1, \mathrm{v} 2) 1 \mathrm{Se}|\mathrm{G}|(\mathrm{v} 3, \mathrm{v} 4) 1 \mathrm{Se}>+<(\mathrm{Sub} 2) 1 \mathrm{Se} \mid(\mathrm{Sub} 1) 1 \mathrm{Se}>+$ $<(\mathrm{Sub} 2) 1 \mathrm{Se}|\mathrm{G}|(\mathrm{Sub} 1) 1 \mathrm{Se}><(\mathrm{v} 1, \mathrm{v} 2) 1 \mathrm{Se}|(\mathrm{v} 3, \mathrm{~V} 4) 1 \mathrm{Se}\rangle+$ $\operatorname{SQRT}(1 / 8) \quad \mathrm{A}(\mathrm{k}=0) \quad\langle(2 \mathrm{~s}, \mathrm{~V} 1) 1 \mathrm{~L}|\mathrm{G}| \quad(1 \mathrm{~s}, \mathrm{v} 3) 1 \mathrm{~L}\rangle \quad\langle\mathrm{V} 2 \mid \mathrm{V} 4\rangle+$ $\operatorname{SQRT}(9 / 8) \quad A(k=0) \quad\langle(2 \mathrm{~s}, \mathrm{v} 1) 3 \mathrm{~L}|\mathrm{G}| \quad(1 \mathrm{~s}, \mathrm{v} 3) 3 \mathrm{~L}\rangle \quad\langle\mathrm{v} 2 \mid \mathrm{v} 4\rangle$

Block (3X1) : < \{(Sub3)3Se, (v1,v2)3Se\}1Se | G | \{(Sub1)1Se, (v3,v4)1Se\} 1Se > = $\operatorname{SQRT}(3 / 8) \quad \mathrm{A}(\mathrm{k}=0) \quad\langle(2 \mathrm{~s}, \mathrm{v} 1) 1 \mathrm{~L}|\mathrm{G}| \quad(1 \mathrm{~s}, \mathrm{v} 3) 1 \mathrm{~L}\rangle \quad\langle\mathrm{V} 2 \mid \mathrm{v} 4\rangle+$

$-\operatorname{SeRT}(3 / 8) \quad \mathrm{A}(\mathrm{k}=0) \quad\langle(2 \mathrm{~s}, \mathrm{v} 1) 3 \mathrm{~L}|\mathrm{G}| \quad(1 \mathrm{~s}, \mathrm{v} 3) 3 \mathrm{~L}\rangle \quad\langle\mathrm{V} 2 \mid \mathrm{v} 4\rangle$

Block $(4 \mathrm{X} 1):<\{(\mathrm{Sub} 4) 1 \mathrm{Po},(\mathrm{v} 1, \mathrm{v} 2) 1 \mathrm{Po}\} 1 \mathrm{Se}|\mathrm{G}|\{(\mathrm{Sub} 1) 1 \mathrm{Se},(\mathrm{v} 3, \mathrm{~V} 4) 1 \mathrm{Se}\} 1 \mathrm{Se}>=$ $-\operatorname{SQRT}(9 / 8) \quad \mathrm{A}(\mathrm{k}=1) \quad\langle(2 \mathrm{p}, \mathrm{V} 1) 1 \mathrm{~L}|\mathrm{G}| \quad(1 \mathrm{~s}, \mathrm{v} 3) 1 \mathrm{~L}\rangle \quad\langle\mathrm{v} 2 \mid \mathrm{v} 4\rangle+$

$-\operatorname{SQRT}(81 / 8) \quad \mathrm{A}(\mathrm{k}=1) \quad\langle(2 \mathrm{p}, \mathrm{v} 1) 3 \mathrm{~L} \quad|\mathrm{G}| \quad(1 \mathrm{~s}, \mathrm{v} 3) 3 \mathrm{~L}\rangle \quad\langle\mathrm{v} 2 \mid \mathrm{v} 4\rangle$

Block $(5 \mathrm{X} 1):<\{(\mathrm{Sub} 5) 3 \mathrm{Po},(\mathrm{v} 1, \mathrm{v} 2) 3 \mathrm{Po}\} 1 \mathrm{Se}|\mathrm{G}|\{(\mathrm{Sub} 1) 1 \mathrm{Se},(\mathrm{v} 3, \mathrm{~V} 4) 1 \mathrm{Se}\} 1 \mathrm{Se}>=$ $-\operatorname{SQRT}(27 / 8) \quad \mathrm{A}(\mathrm{k}=1) \quad\langle(2 \mathrm{p}, \mathrm{v} 1) 1 \mathrm{~L}|\mathrm{G}| \quad(1 \mathrm{~s}, \mathrm{v} 3) 1 \mathrm{~L}\rangle \quad\langle\mathrm{v} 2 \mid \mathrm{v} 4\rangle \quad+$ $\operatorname{SQRT}(27 / 8) \quad \mathrm{A}(\mathrm{k}=1) \quad\langle(2 \mathrm{p}, \mathrm{v} 1) 3 \mathrm{~L}|\mathrm{G}| \quad(1 \mathrm{~s}, \mathrm{v} 3) 3 \mathrm{~L}\rangle \quad\langle\mathrm{v} 2 \mid \mathrm{v} 4\rangle$

Block (2X2) : < \{(Sub2)1Se, (v1,v2)1Se\} 1Se | G | \{(Sub2)1Se, (v3,v4)1Se\} 1Se > = $<(\mathrm{v} 1, \mathrm{v} 2) 1 \mathrm{Se}|\mathrm{G}|(\mathrm{v} 3, \mathrm{v} 4) 1 \mathrm{Se}>+<(\mathrm{Sub} 2) 1 \mathrm{Se} \mid(\mathrm{Sub} 2) 1 \mathrm{Se}>+$

$<(\mathrm{Sub} 2) 1 \mathrm{Se}|\mathrm{G}|(\mathrm{Sub} 2) 1 \mathrm{Se}>\langle(\mathrm{v} 1, \mathrm{v} 2) 1 \mathrm{Se} \mid(\mathrm{v} 3, \mathrm{v} 4) 1 \mathrm{Se}\rangle+$

$1 / 4 \mathrm{~A}(\mathrm{k}=0) \quad\langle(1 \mathrm{~s}, \mathrm{~V} 1) 1 \mathrm{~L}|\mathrm{G}| \quad(1 \mathrm{~s}, \mathrm{v} 3) 1 \mathrm{~L}\rangle \quad\langle\mathrm{V} 2 \mid \mathrm{V} 4\rangle \quad+$

$3 / 4 \mathrm{~A}(\mathrm{k}=0) \quad\langle(1 \mathrm{~s}, \mathrm{v} 1) 3 \mathrm{~L}|\mathrm{G}|(1 \mathrm{~s}, \mathrm{v} 3) 3 \mathrm{~L}\rangle \quad\langle\mathrm{V} 2 \mid \mathrm{V} 4\rangle+$

$1 / 4 \mathrm{~A}(\mathrm{k}=0) \quad\langle(2 \mathrm{~s}, \mathrm{~V} 1) 1 \mathrm{~L}|\mathrm{G}|(2 \mathrm{~s}, \mathrm{~V} 3) 1 \mathrm{~L}\rangle \quad\langle\mathrm{V} 2 \mid \mathrm{V} 4\rangle+$

$3 / 4 \quad A(k=0) \quad\langle(2 \mathrm{~s}, \mathrm{~V} 1) 3 \mathrm{~L}|\mathrm{G}| \quad(2 \mathrm{~s}, \mathrm{~V} 3) 3 \mathrm{~L}\rangle \quad\langle\mathrm{V} 2 \mid \mathrm{V} 4\rangle$

Block $(3 \times 2):<\{(\mathrm{Sub} 3) 3 \mathrm{Se},(\mathrm{v} 1, \mathrm{v} 2) 3 \mathrm{Se}\} 1 \mathrm{Se}|\mathrm{G}|\{(\mathrm{Sub} 2) 1 \mathrm{Se},(\mathrm{v} 3, \mathrm{v} 4) 1 \mathrm{Se}\} 1 \mathrm{Se}>=$ $-\operatorname{SQRT}(3 / 16) \quad \mathrm{A}(\mathrm{k}=0) \quad\langle(1 \mathrm{~s}, \mathrm{v} 1) 1 \mathrm{~L}|\mathrm{G}|(1 \mathrm{~s}, \mathrm{v} 3) 1 \mathrm{~L}\rangle \quad\langle\mathrm{v} 2 \mid \mathrm{v} 4\rangle+$ $\operatorname{SQRT}(3 / 16) \quad A(k=0) \quad<(1 \mathrm{~s}, \mathrm{~V} 1) 3 \mathrm{~L}|\mathrm{G}| \quad(1 \mathrm{~s}, \mathrm{~V} 3) 3 \mathrm{~L}\rangle \quad\langle\mathrm{V} 2 \mid \mathrm{V} 4\rangle+$ $\operatorname{SQRT}(3 / 16) \quad A(k=0) \quad<(2 \mathrm{~s}, \mathrm{~V} 1) 1 \mathrm{~L}|\mathrm{G}| \quad(2 \mathrm{~s}, \mathrm{v} 3) 1 \mathrm{~L}\rangle \quad\langle\mathrm{V} 2 \mid \mathrm{v} 4\rangle+$ $-\operatorname{SQRT}(3 / 16) \quad A(k=0) \quad<(2 \mathrm{~s}, \mathrm{~V} 1) 3 \mathrm{~L} \quad|\mathrm{G}| \quad(2 \mathrm{~s}, \mathrm{~V} 3) 3 \mathrm{~L}>\quad\langle\mathrm{V} 2| \mathrm{V} 4>$

Block $(4 \mathrm{X} 2):<\{(\mathrm{Sub} 4) 1 \mathrm{Po},(\mathrm{v} 1, \mathrm{v} 2) 1 \mathrm{PO}\} 1 \mathrm{Se}|\mathrm{G}|\{(\mathrm{Sub} 2) 1 \mathrm{Se},(\mathrm{v} 3, \mathrm{v} 4) 1 \mathrm{Se}\} 1 \mathrm{Se}>=$ $-3 / 4 A(\mathrm{k}=1) \quad<(2 \mathrm{p}, \mathrm{V} 1) 1 \mathrm{~L} \quad|\mathrm{G}| \quad(2 \mathrm{~s}, \mathrm{~V} 3) 1 \mathrm{~L}\rangle \quad\langle\mathrm{V} 2 \mid \mathrm{V} 4\rangle \quad+$

$-9 / 4 \mathrm{~A}(\mathrm{k}=1) \quad<(2 \mathrm{p}, \mathrm{v} 1) 3 \mathrm{~L} \quad|\mathrm{G}| \quad(2 \mathrm{~s}, \mathrm{v} 3) 3 \mathrm{~L}\rangle \quad\langle\mathrm{v} 2 \mid \mathrm{V} 4\rangle$

Block (5X2) : < (Sub5)3Po, (v1, v2) 3Po $1 \mathrm{Se}|\mathrm{G}|\{(\mathrm{Sub} 2) 1 \mathrm{Se},(\mathrm{v} 3, \mathrm{~V} 4) 1 \mathrm{Se}\} 1 \mathrm{Se}>=$ $-\operatorname{SQRT}(27 / 16) \quad \mathrm{A}(\mathrm{k}=1) \quad<(2 \mathrm{p}, \mathrm{v} 1) 1 \mathrm{~L}|\mathrm{G}| \quad(2 \mathrm{~s}, \mathrm{v} 3) 1 \mathrm{~L}\rangle \quad\langle\mathrm{v} 2 \mid \mathrm{v} 4\rangle+$

$\operatorname{SQRT}(27 / 16) \quad A(k=1) \quad<(2 \mathrm{p}, \mathrm{V} 1) 3 \mathrm{~L}|\mathrm{G}| \quad(2 \mathrm{~s}, \mathrm{~V} 3) 3 \mathrm{~L}\rangle \quad\langle\mathrm{V} 2 \mid \mathrm{V} 4\rangle$

Block $(3 \times 3):<\{(\mathrm{Sub} 3) 3 \mathrm{Se},(\mathrm{v} 1, \mathrm{v} 2) 3 \mathrm{Se}\} 1 \mathrm{Se}|\mathrm{G}|\{(\mathrm{Sub} 3) 3 \mathrm{Se},(\mathrm{v} 3, \mathrm{~V} 4) 3 \mathrm{Se}\} 1 \mathrm{Se}>=$ $<(\mathrm{v} 1, \mathrm{v} 2) 3 \mathrm{Se}|\mathrm{G}|(\mathrm{v} 3, \mathrm{v} 4) 3 \mathrm{Se}><(\mathrm{Sub} 3) 3 \mathrm{Se} \mid$ (Sub3) 3Se $>+$

$<(\mathrm{Sub3}) 3 \mathrm{Se}|\mathrm{G}|$ (Sub3) $3 \mathrm{Se}><(\mathrm{v} 1, \mathrm{~V} 2) 3 \mathrm{Se}|(\mathrm{v} 3, \mathrm{~V} 4) 3 \mathrm{Se}\rangle+$

$3 / 4 \mathrm{~A}(\mathrm{k}=0) \quad<(1 \mathrm{~s}, \mathrm{v} 1) 1 \mathrm{~L}|\mathrm{G}| \quad(1 \mathrm{~s}, \mathrm{v} 3) 1 \mathrm{~L}\rangle \quad\langle\mathrm{V} 2 \mid \mathrm{v} 4\rangle+$

$1 / 4 \mathrm{~A}(\mathrm{k}=0) \quad\langle(1 \mathrm{~s}, \mathrm{~V} 1) 3 \mathrm{~L}|\mathrm{G}| \quad(1 \mathrm{~s}, \mathrm{v} 3) 3 \mathrm{~L}\rangle \quad\langle\mathrm{V} 2 \mid \mathrm{V} 4\rangle+$

$3 / 4 \mathrm{~A}(\mathrm{k}=0) \quad<(2 \mathrm{~s}, \mathrm{~V} 1) 1 \mathrm{~L}|\mathrm{G}| \quad(2 \mathrm{~s}, \mathrm{v} 3) 1 \mathrm{~L}\rangle \quad\langle\mathrm{V} 2 \mid \mathrm{V} 4\rangle+$

$1 / 4 \mathrm{~A}(\mathrm{k}=0) \quad<(2 \mathrm{~s}, \mathrm{~V} 1) 3 \mathrm{~L}|\mathrm{G}| \quad(2 \mathrm{~s}, \mathrm{~V} 3) 3 \mathrm{~L}>\quad<\mathrm{V} 2 \mid \mathrm{V} 4>$ 


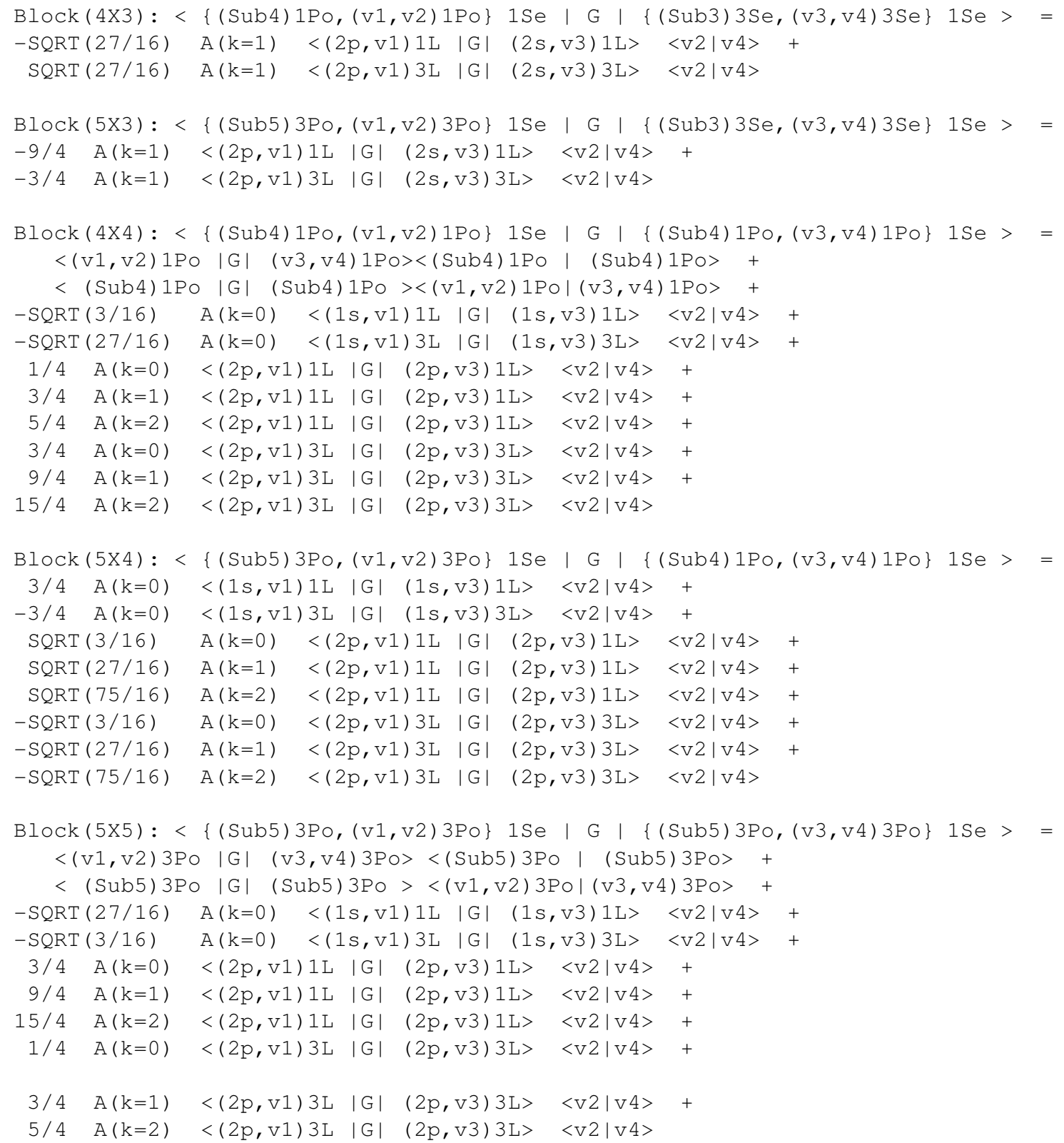

Appendix B. Expanded expressions for the interactions within the $\left|\left\{(1 s, 1 s){ }^{1} S,\left(v_{1} l_{1}, v_{2} l_{2}\right){ }^{1} S\right\}{ }^{1} S\right\rangle$ symbolic block

INTERACTION BLOCKS FOR G CONSERVING L, S

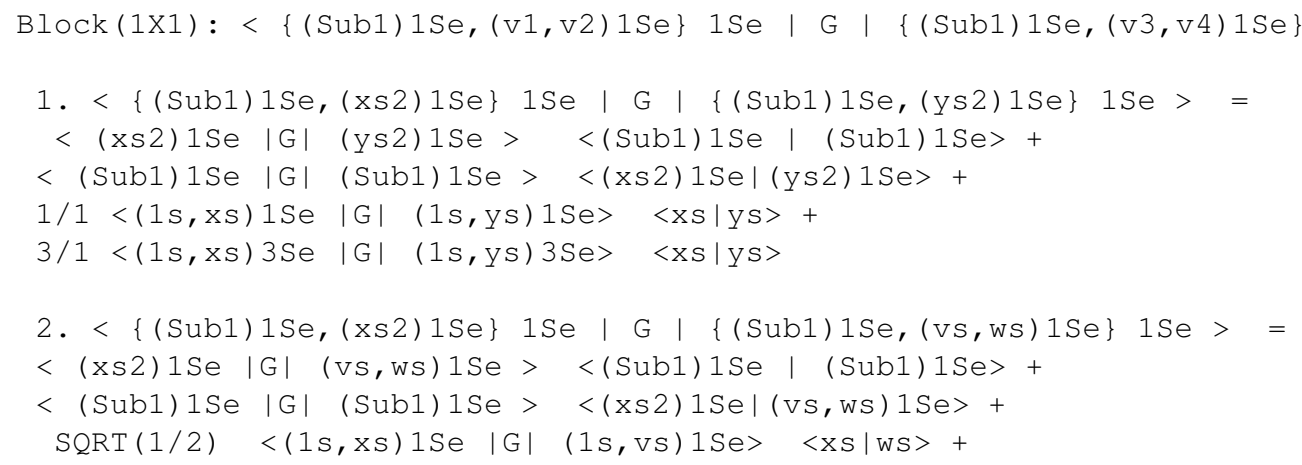




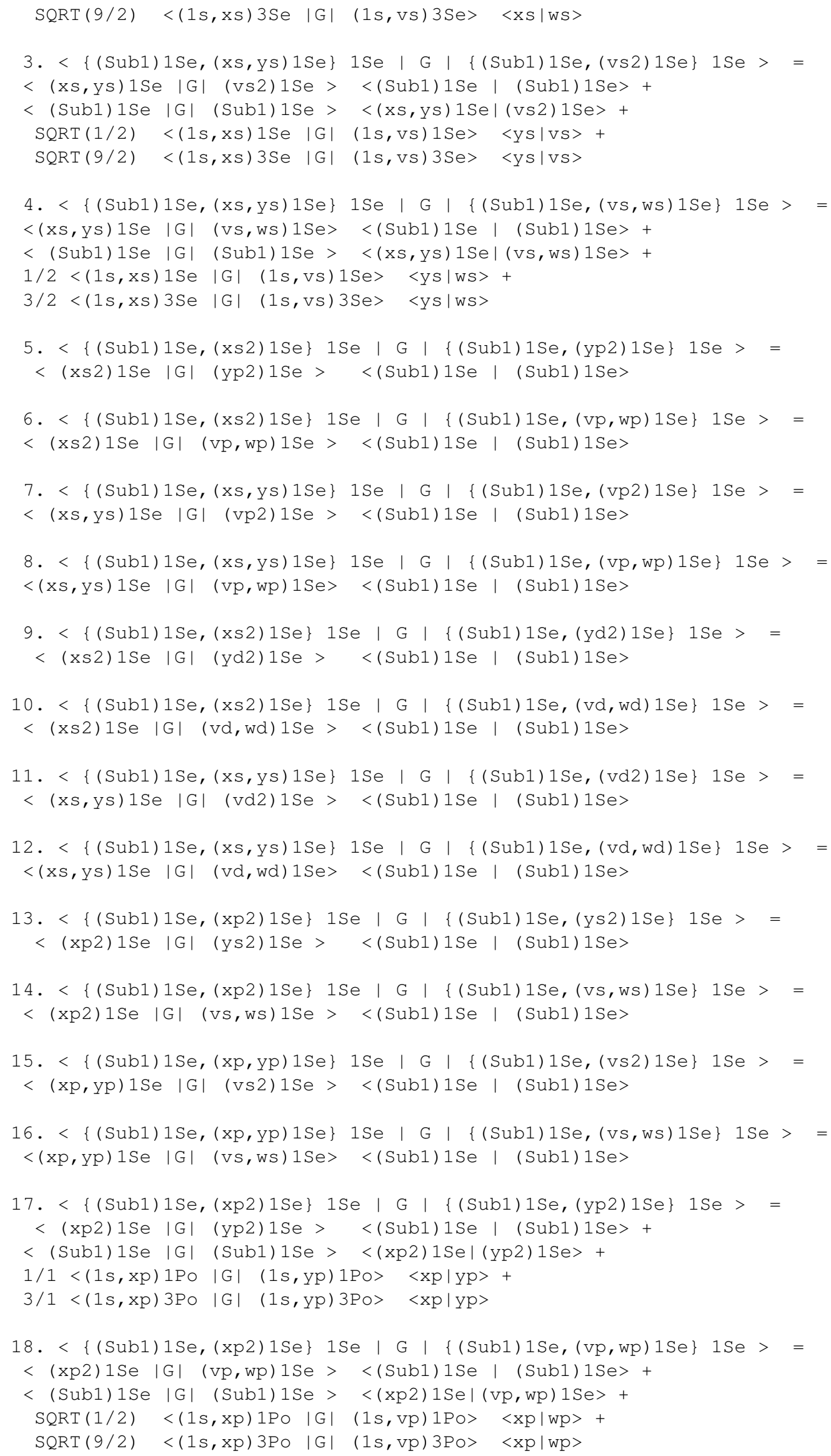


19. < \{(Sub1)1Se, (xp, yp) 1Se\} 1Se | G | \{(Sub1)1Se, (vp2)1Se\} 1Se > =

$<(\mathrm{xp}, \mathrm{yp}) 1 \mathrm{Se}|\mathrm{G}|(\mathrm{vp} 2) 1 \mathrm{Se}>\quad<(\mathrm{Sub} 1) 1 \mathrm{Se} \mid$ (Sub1) 1Se $>+$

$<$ (Sub1)1Se $|\mathrm{G}|$ (Sub1) 1Se > <(xp,yp) 1Se|(vp2)1Se $>+$

$\operatorname{SQRT}(1 / 2) \quad\langle(1 \mathrm{~s}, \mathrm{xp}) 1 \mathrm{PO}|\mathrm{G}| \quad(1 \mathrm{~s}, \mathrm{vp}) 1 \mathrm{Po}\rangle \quad\langle\mathrm{yp} \mid \mathrm{vp}\rangle+$

$\operatorname{SQRT}(9 / 2) \quad\langle(1 \mathrm{~s}, \mathrm{xp}) 3 \mathrm{PO}|\mathrm{G}|(1 \mathrm{~s}, \mathrm{vp})$ 3Po $>\quad \mathrm{yp} \mid \mathrm{vp}\rangle$

20. < \{(Sub1)1Se, (xp, yp) 1Se $\} 1 \mathrm{Se}|\mathrm{G}|\{(\mathrm{Sub} 1) 1 \mathrm{Se},(\mathrm{vp}, \mathrm{wp}) 1 \mathrm{Se}\} 1 \mathrm{Se}>=$

$<(x p, y p) 1 \mathrm{Se}|\mathrm{G}| \quad(\mathrm{vp}, \mathrm{wp}) 1 \mathrm{Se}>\quad<(\mathrm{Sub} 1) 1 \mathrm{Se} \mid$ (Sub1)1Se $>+$

$<(\mathrm{Sub} 1) 1 \mathrm{Se}|\mathrm{G}| \quad(\mathrm{Sub} 1) 1 \mathrm{Se}>\quad<(\mathrm{xp}, \mathrm{yp}) 1 \mathrm{Se} \mid(\mathrm{vp}, \mathrm{wp}) 1 \mathrm{Se}>+$

$1 / 2<(1 \mathrm{~s}, \mathrm{xp}) 1 \mathrm{Po}|\mathrm{G}|(1 \mathrm{~s}, \mathrm{vp}) 1 \mathrm{Po}\rangle \quad\langle\mathrm{yp} \mid \mathrm{wp}\rangle+$

$3 / 2<(1 s, x p) 3 P \circ|G| \quad(1 s, v p) 3 P o>\quad\langle y p| w p>$

21. < \{(Sub1)1Se, (xp2)1Se\} 1Se | G | \{(Sub1)1Se, (yd2)1Se\} $1 \mathrm{Se}>=$ $<(\mathrm{xp} 2) 1 \mathrm{Se}|\mathrm{G}|(\mathrm{yd} 2) 1 \mathrm{Se}>\quad<(\mathrm{Sub} 1) 1 \mathrm{Se} \mid$ (Sub1) 1Se>

22. < \{(Sub1)1Se, (xp2)1Se\} 1Se | G | \{(Sub1)1Se, (vd, wd $) 1 \mathrm{Se}\} 1 \mathrm{Se}>=$ $<(\mathrm{xp} 2) 1 \mathrm{Se}|\mathrm{G}|(\mathrm{vd}$, wd) $1 \mathrm{Se}>\quad\langle(\mathrm{Sub} 1) 1 \mathrm{Se}|$ (Sub1)1Se $>$

23. < \{(Sub1)1Se, (xp,yp) 1Se $\} 1 \mathrm{Se}|\mathrm{G}|\{(\mathrm{Sub} 1) 1 \mathrm{Se},(\mathrm{vd} 2) 1 \mathrm{Se}\} 1 \mathrm{Se}>=$ $<(\mathrm{xp}, \mathrm{yp}) 1 \mathrm{Se}|\mathrm{G}| \quad(\mathrm{vd} 2) 1 \mathrm{Se}>\quad<(\mathrm{Sub} 1) 1 \mathrm{Se} \mid$ (Sub1)1Se $>$

24. < \{(Sub1)1Se, (xp,yp) 1Se $\} 1 \mathrm{Se}|\mathrm{G}|\{(\mathrm{Sub} 1) 1 \mathrm{Se},(\mathrm{vd}$, wd $) 1 \mathrm{Se}\} 1 \mathrm{Se}>=$ $<(x p, y p) 1 \mathrm{Se}|\mathrm{G}| \quad(\mathrm{vd}$, wd) 1Se $>\quad<(\mathrm{Sub} 1) 1 \mathrm{Se} \mid$ (Sub1) 1Se $>$

25. < \{(Sub1)1Se, (xd2) 1Se $1 \mathrm{Se}|\mathrm{G}|\{(\mathrm{Sub} 1) 1 \mathrm{Se},(\mathrm{ys} 2) 1 \mathrm{Se}\} 1 \mathrm{Se}>=$ $<(\mathrm{xd} 2) 1 \mathrm{Se}|\mathrm{G}|$ (ys2)1Se $>\quad<(\mathrm{Sub} 1) 1 \mathrm{Se} \mid$ (Sub1) 1Se $>$

26. < \{(Sub1)1Se, (xd2)1Se\} 1Se | G | \{(Sub1)1Se, (vs, ws)1Se $1 \mathrm{Se}>=$ $<(\mathrm{xd} 2) 1 \mathrm{Se}|\mathrm{G}|$ (vs,ws) $1 \mathrm{Se}>\quad<(\mathrm{Sub} 1) 1 \mathrm{Se} \mid$ (Sub1)1Se>

27. < $\{(\mathrm{Sub} 1) 1 \mathrm{Se},(\mathrm{xd}, \mathrm{yd}) 1 \mathrm{Se}\} 1 \mathrm{Se}|\mathrm{G}|\{(\mathrm{Sub} 1) 1 \mathrm{Se},(\mathrm{vs} 2) 1 \mathrm{Se}\} 1 \mathrm{Se}>=$ $<(\mathrm{xd}, \mathrm{yd}) 1 \mathrm{Se}|\mathrm{G}|$ (vs2)1Se > <(Sub1)1Se | (Sub1)1Se>

28. < \{(Sub1)1Se, (xd, yd) 1Se $\} 1 \mathrm{Se}|\mathrm{G}|\{(\mathrm{Sub} 1) 1 \mathrm{Se},(\mathrm{vs}, \mathrm{ws}) 1 \mathrm{Se}\} 1 \mathrm{Se}>=$ $<(\mathrm{xd}, \mathrm{yd}) 1 \mathrm{Se}|\mathrm{G}|$ (vs, ws) 1Se $>\quad$ (Sub1) 1Se | (Sub1)1Se>

29. < \{(Sub1)1Se, (xd2) 1Se $\} 1 \mathrm{Se}|\mathrm{G}|\{(\mathrm{Sub} 1) 1 \mathrm{Se},(y p 2) 1 \mathrm{Se}\} 1 \mathrm{Se}>=$ $<(\mathrm{xd} 2) 1 \mathrm{Se}|\mathrm{G}|$ (yp2)1Se $>\quad<(\mathrm{Sub} 1) 1 \mathrm{Se} \mid$ (Sub1)1Se>

30. < \{(Sub1)1Se, (xd2) 1Se\} 1Se | G | \{(Sub1)1Se, (vp, wp) 1Se $\} 1 \mathrm{Se}>=$ $<(\mathrm{xd} 2) 1 \mathrm{Se}|\mathrm{G}|(\mathrm{vp}, \mathrm{wp}) 1 \mathrm{Se}>\quad$ (Sub1) 1Se | (Sub1)1Se>

31. < $\{(\mathrm{Sub} 1) 1 \mathrm{Se},(\mathrm{xd}, \mathrm{yd}) 1 \mathrm{Se}\} 1 \mathrm{Se}|\mathrm{G}|\{(\mathrm{Sub} 1) 1 \mathrm{Se},(\mathrm{vp} 2) 1 \mathrm{Se}\} 1 \mathrm{Se}>=$ $<(\mathrm{xd}, \mathrm{yd}) 1 \mathrm{Se}|\mathrm{G}|(\mathrm{vp} 2) 1 \mathrm{Se}>\quad<(\mathrm{Sub} 1) 1 \mathrm{Se} \mid$ (Sub1)1Se>

32. < \{(Sub1)1Se, (xd, yd) 1Se $\} 1 \mathrm{Se}|\mathrm{G}|\{(\mathrm{Sub} 1) 1 \mathrm{Se},(\mathrm{vp}, \mathrm{wp}) 1 \mathrm{Se}\} 1 \mathrm{Se}>=$ $<(x d, y d) 1 \mathrm{Se}|\mathrm{G}| \quad(\mathrm{vp}, \mathrm{wp}) 1 \mathrm{Se}>\quad<(\mathrm{Sub} 1) 1 \mathrm{Se} \mid$ (Sub1) 1Se>

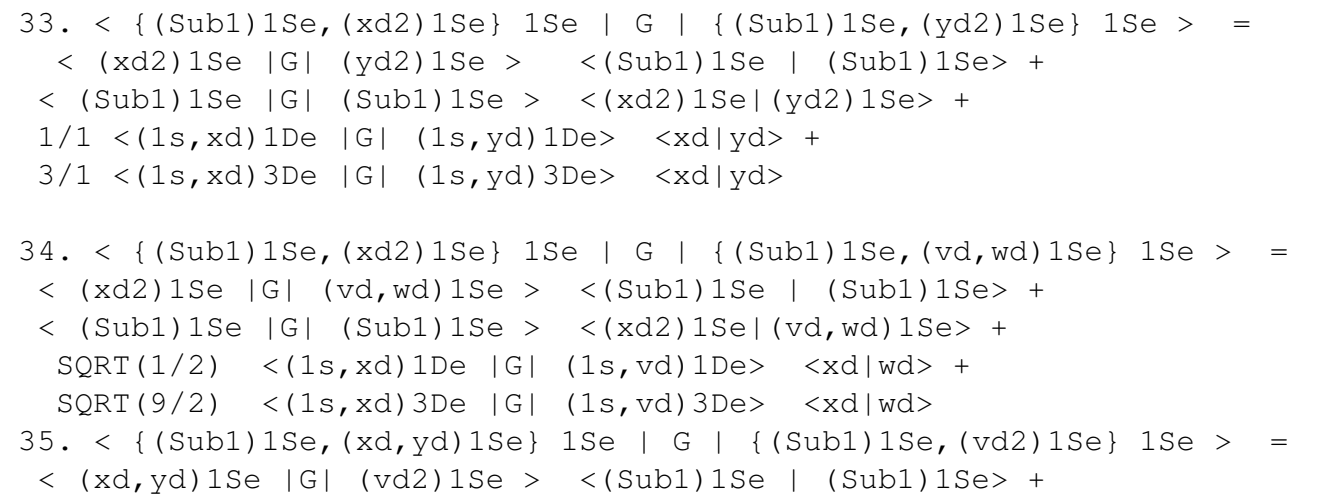




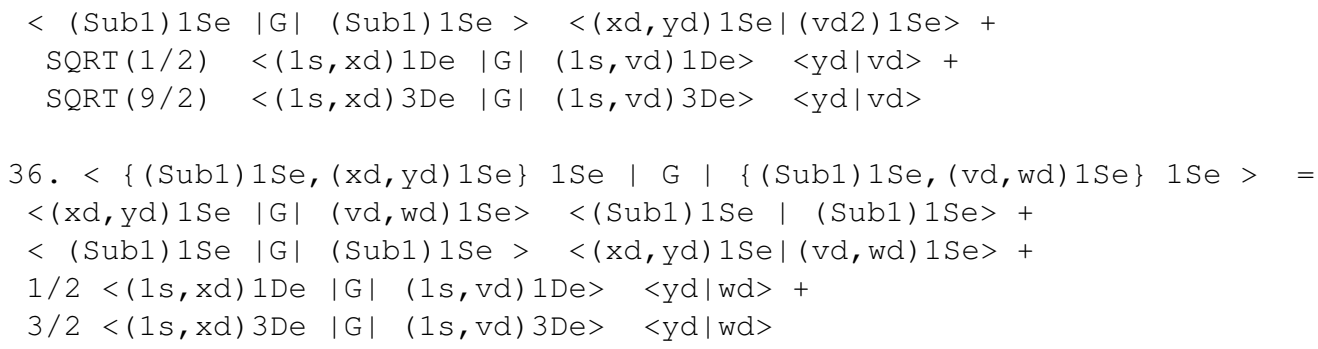

\section{Acknowledgement}

This work was supported by the Chemical Sciences, Geosciences, and Biosciences Division, Office of Basic Energy Sciences, Office of Science, US Department of Energy.

\section{References}

[1] J.C. Slater, Phys. Rev. 34, 1293; 1312 (1929).

[2] D.R. Hartree, W. Hartree, and B. Swirles, Philos. Trans. A 238, 229 (1939).

[3] A.P. Jucys, Proc. Roy. Soc. London Ser. A 173, 59 (1939).

[4] C. Froese Fischer, Douglas Rayner Hartree: His Life in Science and Computing (World Scientific, Singapore, 2004).

[5] A. Porter, Mem. Proc. Manch. Lit. Phil. Soc. 79, 75 (1934-1935).

[6] G. Racah, Phys. Rev. 61, 106 (1942); 62, 438 (1942); 63, 368 (1943).

[7] R. Karazija (private communication).

[8] A. Hibbert, Comput. Phys. Commun. 1, 359 (1970); 2, 180 (1971); 7, 318 (1974); 8, 329 (1974).

[9] C. Froese Fischer, Comput. Phys. Commun. 1, 151 (1969); 4, 107 (1972); 14, 145 (1978); 64, 431 (1991).

[10] A. Bar-Shalom and M. Klapisch, Comput. Phys. Commun. 50, 375 (1988).
[11] G. Gaigalas, Z. Rudzikas, and C. Froese Fischer, J. Phys. B 30, 3747 (1997).

[12] Z. Rudzikas, Theoretical Atomic Spectroscopy (Cambridge University Press, Cambridge, 1997).

[13] C. Froese Fischer and B. Liu, Comput. Phys. Commun. 64, 406 (1991).

[14] L. Sturesson and C. Froese Fischer, Comput. Phys. Commun. 74, 432 (1993).

[15] P. Jönsson (1996) (unpublished).

[16] I.P. Grant and H. Quiney, Mol. Phys. (submitted).

[17] A. Weiss (1995) (private communication).

[18] O. Zatsarinny and C. Froese Fischer, Comput. Phys. Commun. 124, 247 (2000).

[19] C. Froese Fischer, Int. J. Supercomputer Appl. 5, 5 (1991).

[20] E.R. Davidson, J. Comput. Phys. 17, 87 (1975); A. Stathopoulos and C. Froese Fischer, Comput. Phys. Commun. 79, 268 (1994).

[21] A. Irimia and C. Froese Fischer, Physica Scripta (2004) (accepted).

[22] A.W. Weiss, Phys. Rev. A 51, 1067 (1995).

[23] R. Matulioniene, Angular momentum algebra for symbolic state expansion in atomic theory, $\mathrm{PhD}$ thesis (University of Toledo, 1999).

[24] A.P. Jucys and A.A. Bandzaitis, The Theory of Angular Momentum in Quantum Mechanics, 2nd edn. (Mokslas, Vilnius, 1977).

\title{
KAMPINIS INTEGRAVIMAS PANAUDOJANT SIMBOLINIUS BŪSENŲ SKLEIDINIUS
}

\author{
C. Froese Fischer ${ }^{a}$, D. Ellis ${ }^{b}$ \\ ${ }^{\text {a }}$ Vanderbilto universitetas, Nešvilis, JAV \\ ${ }^{\mathrm{b}}$ Toledo universitetas, Toledas, JAV
}

\section{Santrauka}

Pateiktas bendras algoritmas atomo banginès funkcijos skleidiniui gauti ir įvairių atominių dydžių operatorių matriciniams elementams skaičiuoti. Elektrostatinès sąveikos operatoriaus matri- cinis elementas yra išreiškiamas kaip trijų daugiklių - konstantos, dvielektronio matricinio elemento ir daugiklio, priklausančio nuo sąveikaujančių elektronų orbitinių kvantinių skaičių, - sandauga. Išsamiai aprašyta berilio $1 s^{2} 2 s^{2}{ }^{1} S$ pagrindinè būsena. 